\title{
Stress-Optical Coefficient of the Compatible Poly(styrene)- Poly(methyl methacrylate)-Benzene Solution System
}

\author{
Tai Jun LIM, Hee Tai Chung, and In Jae ChUNG* \\ Department of Chemical Engineering, \\ Korea Advanced Institute of Science and Technology, \\ P. O. Box 131, Cheongryang, Seoul, 130-650, Korea
}

(Received March 16, 1989)

\begin{abstract}
A ternary solution of poly(styrene)-poly(methyl methacrylate)-benzene has been studied rheo-optically. Shear viscosity of the PS-PMMA solutions measured by a capillary measuring apparatus is well fitted by the Hashin's blending rule. Flow birefringence $\Delta n$ and orientation angle $\chi$ of the solution in the steady shear flow is measured by the phase modulated flow birefringence (PMFB) technique. By comparing the shear viscosities measured in a capillary apparatus with the optical data from the PMFB, the stress-optical coefficients of the solution and of the pure PS and PMMA solutions are obtained. In a blend state, the value of stress-optical coefficient is able to be predicted as a simple linear additive relation with the values of two pure polymer species and the compositions. The stress-optical law is turned out to be applicable to compatible PS-PMMA-benzene solution system.
\end{abstract}

KEY WORDS Ternary Solution / Poly(styrene)-Poly(methyl methacrylate)-Benzene / Stress-Optical Law / Stress-Optical Coefficient / Flow Birefringence / Phase Modulated Flow Birefringence /

Polymer blend has been an important role in polymer science and technology because of its superior properties rather than those of homopolymers. The concept of physically blending of two existing polymers to obtain new products has not been fully understood. ${ }^{1}$ Especially, an investigation of flow behaviors of the polymer blend system is one of the most interesting problems to be solved. So far, unfortunately, the development of a theoretical consideration for the polymer blends has been a very difficult work because of their complex behaviors. Therefore, an experimental study is an useful tool to understand the rheological properties of the polymer blends.

As one of the measuring techniques for the polymer system, the flow birefringence method has been considered as one of the most productive experimental techniques suitable for the investigation of the rheological properties of the polymeric liquids under a certain deformation. $^{2-5}$ This method can measure some rheological properties of the polymer materials such as the shear stress and the normal stress difference more accurately and easily rather than the mechanical measuring devices which have some shortcomings in measuring the rheological properties because of the inherent compliance of its force transducers. ${ }^{2}$

In order to apply the flow birefringence method to the rheology of the polymer materials, it is necessary to check the applicability of the stress-optical law. ${ }^{6,7}$ This law assumes that in any case the deviatoric components of the stress tensor and those of the refractive index tensor of the polymer chains have a linear relation as follows:

$$
n=C \sigma
$$

\footnotetext{
* To whom all correspondence should be addressed.
} 
where $n$ and $\sigma$ is the refractive index tensor and stress tensor respectively, and $C$ is the stressoptical coefficient. A linear relation between the $n$ and $\sigma$ in a certain flow system of the polymeric liquid indicates a homogeneity in the molecular structures. It is well known that the stress-optical law is valid for a weak flow such as shear flow in which the polymer chains show Gaussian statistics, not only in the steady state $^{8}$ but also in the transient state..$^{3,5}$

In general, for a homopolymer system, the stress-optical coefficient $C$ is a function of the temperature and the kind of the solvent but is independent of the molecular weight and molecular weight distribution. ${ }^{9}$ For a compatible polymer blend system of two different polymer species, however, the effect of the blending of two components on the stress-optical coefficient has not been sufficiently investigated.

So far, there is no explicit result about the flow birefrigence behaviors of the solution blend system of two different polymer species. In this paper, we measured the flow birefringences and orientation angles in the steady state flow of a compatible ternary solution system, poly(styrene)-poly(methyl methacrylate) in benzene. By comparing these optical data with the mechanical data, we obtained the stress-optical coefficients for each blend and checked the applicability of the stress-optical law to these blends.

\section{EXPERIMENTAL}

\section{Materials}

The ternary solution of poly(styrene) and poly(methyl methacrylate) in benzene with various compositions are used as the experiment materials for both. of the optical and mechanical measurement. The blend ratios of the PMMA to PS based on the weights are chosen as $0,20,40,60,80$, and $100 \mathrm{wt} \%$, respectively. Here the blend ratio of $0 \mathrm{wt} \%$ denotes the pure PS solution and $100 \mathrm{wt} \%$ the pure PMMA solution. The weight-average molecular weight $\bar{M}_{w}$ of the pure PS in $2.95 \times 10^{5}$ and that of pure PMMA is $1.7 \times 10^{5}$, measured by Gel Permeation Chromatography. The polydispersity index of PS and PMMA is 2.4 and 3.5, respectively. The solvent, benzene is a good and matching mutual solvent for both of the PS and PMMA, and the refractive index of benzene 1.5 is not much different from the value of 1.6 of PS and 1.55 of PMMA. For these reasons, through the optical measurements, the form birefringence effect can be easily eliminated. The total concentration of PS-PMMA solution used in this study is $7 \mathrm{wt} \%$ and $9.5 \mathrm{wt} \%$, which corresponds to 0.062 and $0.084 \mathrm{~g} \mathrm{~cm}^{-3}$, respectively. The concentration values of the experimental ternary solution are in below of the critical concentration $C^{* *}$ reported by Pierri and Dondos, ${ }^{10}$ above which the incompatibility between the PS and PMMA has been appeared. At a higher concentration than about $12 \mathrm{wt} \%$, the scattering or dispersion of light beam was occurred because of the phase separation of the samples so that the optical measurement was impossible.

\section{Apparatus and Measurements}

To obtain the stress-optical coefficients of the PS-PMMA-benzene ternary solution, both of the capillary measuring apparatus and the PMFB instrument are constructed. In the capillary apparatus, the polymer sample is forced to flow through a capillary (diameter $=$ $0.472 \mathrm{~mm}$ ) by constant pressure and this pressure value is measured by a manometer. With measuring the flow rate of the solution, the shear viscosity $\eta$ can be obtained by the Hagen-Poiseuille equation,

$$
\eta=\frac{\pi \Delta p r^{4}}{8 L Q}
$$

where $\Delta p$ is the pressure difference measured in a manometer, $r$ and $L$ is the radius and length of the capillary, and $Q$ represents the flow rate of the solution. From this relation, the average values of the shear rate $\dot{\gamma}$ and the shear stress $\sigma$ are determined by: 


$$
\langle\dot{\gamma}\rangle=\frac{\Delta p \cdot r}{3 \eta L}, \quad\langle\sigma\rangle=\eta \cdot\langle\dot{\gamma}\rangle
$$

where $\langle\cdots\rangle$ denotes the average value. We derived the eq 3 with an assumption that the velocity profile of the polymer sample in the capillary is fully developed.

Birefringence $\Delta n$ and orientation angle $\chi$ of a polymer blend are measured by the phase modulated flow birefringence PMFB technique. By the PMFB technique we can measure the changes of optical anisotropy with a great sensitivity because it utilizes the changes of phase retradations of the monochromatic light beam travelled through the polymer material. ${ }^{11}$ Also it has an advantage that the variations of the $\Delta n$ and $\chi$ of the polymeric liquids under the deformation can be measured simultaneously in time. Figure 1 shows the diagram of the PMFB system. The detail descriptions of the optical alignment and the theoretical consideration are explained in elsewhere. ${ }^{11,12}$ Polymer sample is inserted in

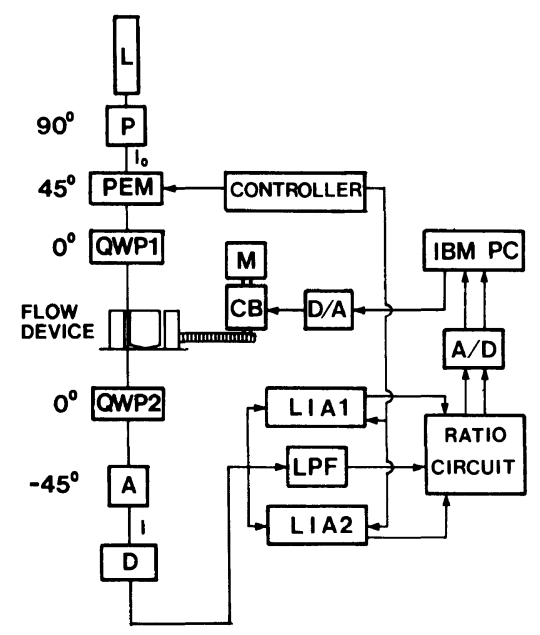

Figure 1. Schematic diagram of the experimental setup of PMFB system: L, He-Ne laser source; P, polarizer; PEM, photoelastic modulator; QWP, quarter wave plate; A, analyzer; D, detector. The numbers listed with the optical components denote the orientation of the optic axis for each component with respect to the flow direction. LIA1 and LIA2 describes the lock-in amplifiers, LPF the low pass filter, and CB the electromagnetic clutch/brake assembly. an annular gap with $1.2 \mathrm{~mm}$ size between the inner and outer cylinder of the coaxial cylinder part in the flow device. The steady shear flow is generated by a computer controlling of the electromagnetic clutch/brake assembly CB. A photoelastic modulator PEM induces a retardation in the phase of the transmitted light beam that oscillates sinusoidally with a resonant frequency $\omega$ of $50.3 \mathrm{kHz}$. With the PEM and the other optical components which have the individual optic axis with respect to the flow direction, the intensity of the laser beam at the photo-detector $\mathrm{D}$ can be expressed by the Jones calculus ${ }^{13}$ as follows:

$$
\begin{aligned}
\dot{I} / I_{0}= & \frac{1}{2}\left[1+\sin 2 \chi \cdot \sin \delta \cdot \cos \delta_{\mathrm{p}}\right. \\
& \left.+\cos 2 \chi \cdot \sin \delta \cdot \sin \delta_{\mathrm{p}}\right]
\end{aligned}
$$

where $\chi$ and $\delta$ is the unknown orientation angle and retardation of the polymer sample in a shear flow, $\delta_{\mathrm{p}}$ represents the induced retardation from the PEM, and $I_{0}$ denotes the intensity of the light beam prior to the PEM. The two lock-in amplifiers tuned to $\omega$ and $2 \omega$ produce the two electric signals $R_{\omega}$ and $R_{2 \omega}$ which contain the component of the first and second harmonics with the resonant frequency of PEM, respectively.

These two signals are acquired simultaneously by an A/D converter, and used to calculate the birefringence and orientation angle of the polymer sample by the following equations:

$$
\begin{aligned}
& \Delta n=-(\lambda / 2 \pi d) \sin ^{-1} \sqrt{R_{\omega}^{2}+R_{2 \omega}^{2}} \\
& \chi=\frac{1}{2} \tan ^{-1}\left(R_{2 \omega} / R_{\omega}\right)
\end{aligned}
$$

where $\lambda$ is the wavelength of the $\mathrm{He}-\mathrm{Ne}$ laser beam $632.8 \mathrm{~nm}$ and $\mathrm{d}$ represents the optical path length $26 \mathrm{~mm}$. All procedures for the control of the flow generation and for the data acquisitions were performed by a personal computer. 


\section{RESULTS AND DISCUSSION}

\section{Composition Dependence on Shear Viscosity}

Figure 2 shows shear viscosities of the PSPMMA-benzene solution with a fixed total concentration of $9.5 \mathrm{wt} \%$ but different blend ratios of PS-PMMA. They are measured in the capillary apparatus at a fixed shear rate. The shear viscosity of pure PS solution is larger than that of pure PMMA solution and the shear viscosity $\eta$ of the blend shows some distributed values between the values of two pure materials. The Hagen-Poiseuille equation used in calculating of the shear viscosity in the capillary apparatus can not accurately measure the $\eta$ of the polymer solution in the high shear rate region. For this reason, in this paper, we mainly consider the zero-shear rate viscosity $\eta_{0}$ which denotes the limiting value at shear rate $\dot{\gamma} \rightarrow 0$ in order to compare the optical data.

Even though not represented in the paper, the shear viscosity of the $7 \mathrm{wt} \%$ solution shows a very similar pattern to that of $9.5 \mathrm{wt} \%$

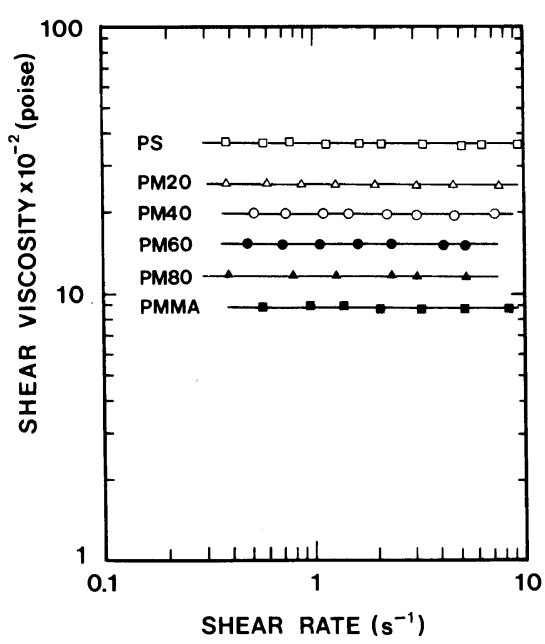

Figure 2. Shear viscosity with the shear rates for the ternary solution with $9.5 \mathrm{wt} \%$ of PS-PMMA. The codes listed in figure denote the blend ratios of PMMA to PS: PS $(\square)$ represents the PS pure solution, PM20 $(\triangle)$,


and $80 \mathrm{wt} \%$ of PMMA with respect to total weight of PS-PMMA, respectively. PMMA ( $\square$ ) denotes the PMMA pure solution. solution, but some lower absolute values of $\eta_{0}$ for each composition. Figure 3 represents the composition dependence of the $\eta_{0}$ for $7 \mathrm{wt} \%$ and $9.5 \mathrm{wt} \%$ solutions. The distribution of $\eta_{0}$ shows some moderate decaying pattern with increase of the PMMA weight fraction. In general, for an incompatible polymer blend system, an occurrence of the viscosity maximum at certain composition is produced. ${ }^{1}$ Figure 3, however, does not show any maximum viscosity in this experimental concentration range. This fact indicates that the PSPMMA solution of $7 \mathrm{wt} \%$ and $9.5 \mathrm{wt} \%$ are in the compatible region like the experimental results of Pierri and Dondos. ${ }^{10}$

It may be interesting to consider the blending rule for the shear viscosities of the ternary solution. For a ternary solution of polymer-1/ polymer-2/solvent, the phase separation make it very difficult to develop a blending rule which predicts accurately the shear viscosities through all concentration ranges. But, for the compatible region, a shear viscosity can be predicted by some blending rules. The blending rules proposed by Hashin ${ }^{14}$ and by Heitmiller ${ }^{15}$ are testified for a ternary solution

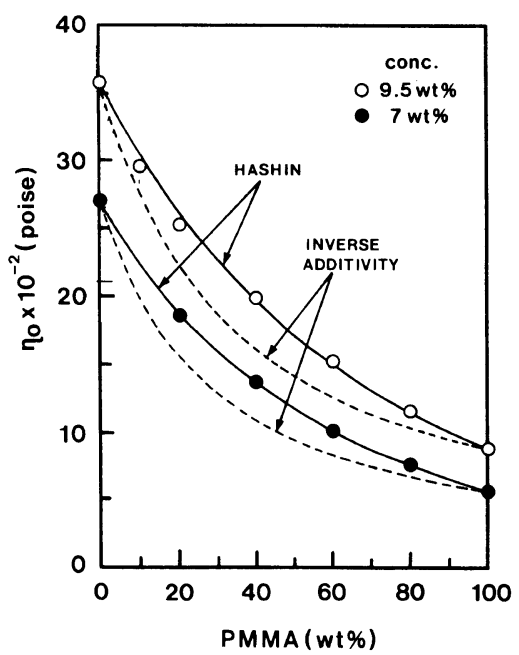

Figure 3. Zero-shear viscosity with the compositions for $9.5 \mathrm{wt} \%(\bigcirc)$ and $7 \mathrm{wt} \%$ (O) of PS-PMMA solution blends: the solid line denotes the theoretical predictions by Hashin $^{12}$ and the dashed line the inverse additivity rule by Heitmiller. ${ }^{13}$ 
of PS/PMMA/benzene. Hashin's rule described as a solid line in Figure 3 is expressed as follows:

$$
\eta_{\mathrm{b}}=\eta_{\mathrm{pm}}+\frac{w}{1 /\left(\eta_{\mathrm{ps}}-\eta_{\mathrm{pm}}\right)+2(1-w) / 5 \eta_{\mathrm{pm}}}
$$

where $\eta_{\mathrm{b}}$ is the $\eta_{\mathrm{o}}$ in blend state and $\eta_{\mathrm{ps}}$ and $\eta_{\mathrm{pm}}$ represents that of the pure PS and PMMA, respectively, and $w$ denotes the blend ratios of the PMMA to PS. The "inverse additivity rule" proposed by Heitmiller $e t$ al. described as a dashed line in Figure 3 has a form as follows:

$$
1 / \eta_{\mathrm{b}}=w / \eta_{\mathrm{ps}}+(1-w) / \eta_{\mathrm{pm}}
$$

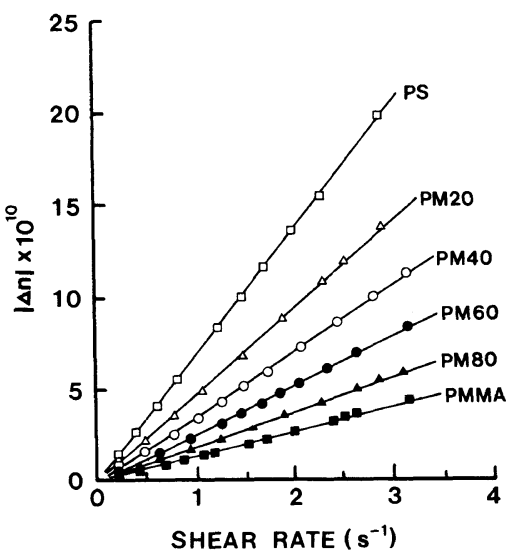

Figure 4. Flow birefringence $\Delta n$ with the shear rates for $9.5 \mathrm{wt} \%$ of PS-PMMA solution blends. Meaning of the codes is explained in caption of Figure 2.

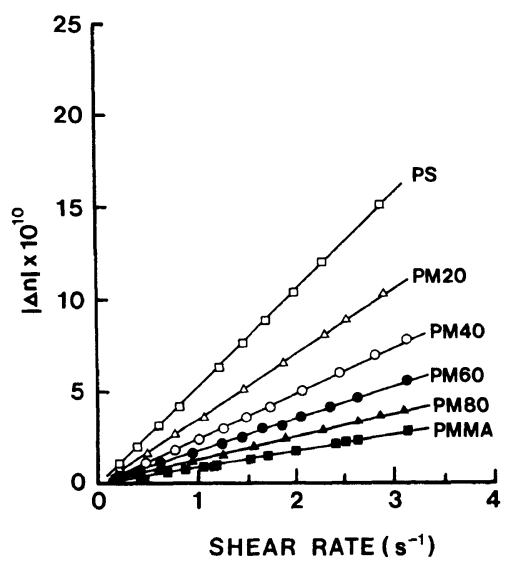

Figure 5. Flow birefringence $\Delta n$ with the shear rates for $7 \mathrm{wt} \%$ of PS-PMMA solution blends. Meaning of the codes is explained in caption of Figure 2.
As shown in Figure 3, the Hashin's rule predicts well the shear viscosity of the blend state, but the "inverse additivity rule" fails to predict it for the compatible PS-PMMA solution.

\section{Birefringences and Orientation Angles}

Flow birefringence $\Delta n$ of the PS-PMMA solution of $9.5 \mathrm{wt}^{\circ} \%$ and $7 \mathrm{wt} \%$ in the steady shear flow is represented in Figures 4 and 5, respectively. For a rather precise analyses of blending effects on the optical properties, we are only concerned about the magnitudes of the birefringence not the signs. Because of the relatively low concentration, the flow birefringences produced by the shear flow increase linearly with the shear rates for each blend in the range of shear rates up to about $3 \mathrm{~s}^{-1}$. But, at the same shear rate, the $\Delta n$ of the $9.5 \mathrm{wt} \%$ solution shows a higher value than that of $7 \mathrm{wt} \%$ solution for all compositions.

The orientation angle of the polymeric liquids in the flow state represents the degree of the average oriented angle of the polymer chains with respect to the flow direction. We represent the orientation angles with the shear

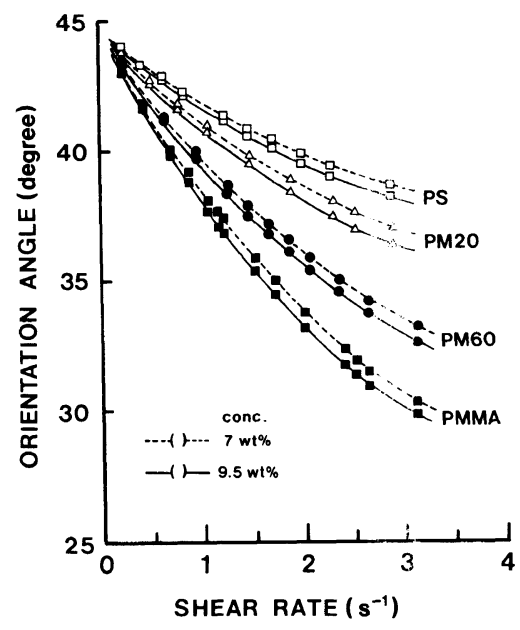

Figure 6. Orientation angle $\chi$ with the shear for $7 \mathrm{wt} \%$ and $9.5 \mathrm{wt} \%$ of PS-PMMA solution blends: dashed lines represent the $\chi$ for $7 \mathrm{wt} \%$ solution and solid lines for $9.5 \mathrm{wt} \%$. Meaning of codes is the sarne as explained in Figure 2. 
rates for both solutions of $7 \mathrm{wt} \%$ and $9.5 \%$ in Figure 6. The solutions of the PS-PMMA and pure materials in benzene show the general pattern of the orientation angle for a polymer solution which has 45 degrees at a static state and decreases with increasing the shear rate. The variation of the PS-PMMA total concentration does not affect significantly the orientation angle.

\section{Stress-Optical Coefficient}

The stress optical law described in eq 1 can be changed to the following form for a simple shear flow:

$$
\frac{\Delta n \sin 2 \chi}{2}=C \cdot \sigma
$$

where $\sigma$ is the shear stress of the polymer sample. By using eq 9, the zero-shear rate viscosity $\eta_{0}$ can be obtained from both of the capillary and optical measurements,

$$
\eta_{0}=\left.\frac{|\Delta n| \cdot \sin 2 \chi}{2 C \dot{\gamma}}\right|_{\dot{\gamma}=0}=\left.\frac{\langle\sigma\rangle}{\dot{\gamma}}\right|_{\dot{\gamma}=0}
$$

The stress-optical coefficient value can be calculated by dividing the terms of $|\Delta n| \cdot \sin 2 \chi / 2 \dot{\gamma}$ with the $\eta_{0}$ from the capillary apparatus. The plots of stress-optical coefficient and zero shear rate viscosity show the same trend in Figure 7.

In Figure 8, absolute values of the stressoptical coefficient for the PS-PMMA solutions are represented with the compositions. There was no dependence of the concentration of the $C$ values between the $7 \mathrm{wt} \%$ and $9.5 \mathrm{wt} \%$. The $C$ values of the pure PS and pure PMMA solutions in benzene are determined as follows:

$\left|C_{\mathrm{ps}}\right|=(9.6 \pm 0.15) \times 10^{-10} \mathrm{~cm}^{2} \mathrm{dyn}^{-1}$

$\left|C_{\mathrm{pm}}\right|=(7.1 \pm 0.2) \times 10^{-10} \mathrm{~cm}^{2} \mathrm{dyn}^{-1}$

The absolute stress-optical coefficient value of the pure PMMA is lower than that of PS, which corresponds to the other research. ${ }^{16}$ However, the stress-optical coefficients of the two pure materials in benzene have some

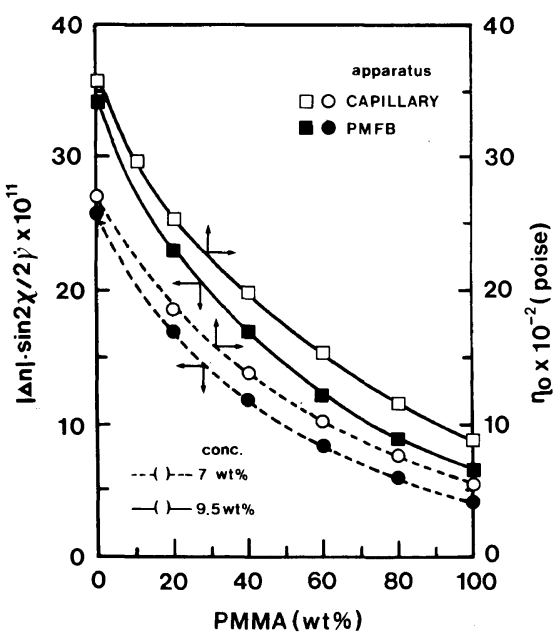

Figure 7. Zero-shear viscosity $\eta_{0}$ measured by the capillary apparatus and its corresponding optical term of $|\Delta n| \cdot \sin 2 \chi / 2 \gamma$ by the PMFB technique with the compositions. Open symbols denote the $\eta_{0}$ by the capillary apparatus: $9.5 \mathrm{wt} \%(\square) ; 7 \mathrm{wt} \%(\bigcirc)$, and closed symbols the optical term from PMFB: $9.5 \mathrm{wt} \%(\mathbf{\square}) ; 7 \mathrm{wt} \%(\boldsymbol{O})$ of PS-PMMA solution blends.

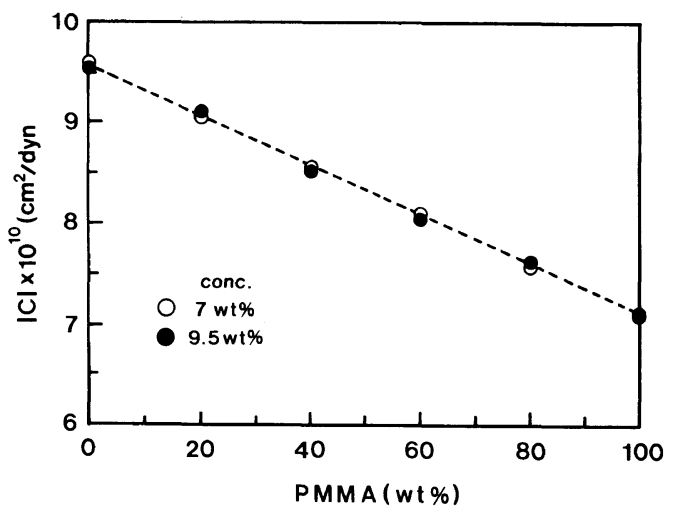

Figure 8. Stress-optical coefficients of the PS-PMMA solutions with the blend ratios of PMMA to PS for concentration of $7 \mathrm{wt} \%(O)$ and $9.5 \mathrm{wt} \%(\bigcirc)$. Dashed line represents the linear relation of $C_{\mathrm{b}}=w \cdot C_{\mathrm{pm}}+$ $(1-w) \cdot C_{\mathrm{ps}}$.

different values from the results of those solution in Aroclor. ${ }^{16}$ This is due to the different roles of solvents, benzene and Aroclor. A similar case has been reported for poly(styrene) in TCP and poly(styrene) in bulk or in Aroclor. ${ }^{17}$

The stress-optical coefficients in the blend 




Figure 9. Plot of $|\Delta n| \cdot \sin 2 \chi / 2 \dot{\gamma} \eta(\dot{\gamma})$ against shear rates for the $9.5 \mathrm{wt} \%$ of PS-PMMA solutions. Meaning of codes is the same as explained in Figure 2.

states show a linear combination of two values of pure PS and PMMA with the compositions. This linear relation depicted as a dashed line in Figure 8 has a form as follows:

$$
C_{\mathrm{b}}=w C_{\mathrm{pm}}+(1-w) C_{\mathrm{ps}}
$$

where $C_{\mathrm{b}}$ is the stress-optical coefficient of the blend state, $C_{\mathrm{pm}}$ and $C_{\mathrm{ps}}$ represents that of PMMA and PS respectively, and $w$ is the blend ratio of PMMA to PS. This linear variation of the $\mathrm{C}$ with the composition for the PS-PMMA solution can be also found for a compatible blend of styrene-butadiene rubbers SBR. ${ }^{16}$ For an incompatible blend of SBR, the variation of the $\mathrm{C}$ shows a non-linear pattern with the composition. As pointed out in the above section, the 7 and $9.5 \mathrm{wt} \%$ of the PSPMMA solutions are in the compatible region. Therefore, the linear variation of the stressoptical coefficient with the composition eq 12 is a meaningful result for the test PS-PMMA solutions.

From these results, we can check the applicability of the stress-optical law for the blend state of the PS-PMMA solutions as follows; If we assume that the stress-optical law does not applicable for the blend state of this system, the term of $|\Delta n| \sin 2 \chi / 2 \dot{\gamma} \eta(\dot{\gamma})$ which corresponds to $C$ as described in eq 9 will not show any constancy with the shear rates. We plotted the term against the shear rates for the $9.5 \mathrm{wt} \%$ solution in Figure 9. For each composition, a good constancy of the term of $|\Delta n| \sin 2 \chi \mid$ $2 i \eta(\dot{\gamma})$ with the shear rates is observed. Consequently, the stress-optical law is proven applicable to the compatihle PS-PMMA solutions.

\section{REFERENCES}

1. D. R. Paul and S. Newman, "Polymer Blends," Vol. 1, Academic Press, Inc., N. Y., 1978, Chapters 1, 7.

2. B. E. Zebrowski and G. G. Fuller, J. Polym. Sci., Polym. Phys. Ed., 23, 575 (1985).

3. K. Osaki, N. Bessho, T. Kojimoto, and M. Kurata, J. Rheol., 23, 457 (1979).

4. K. Osaki, N. Bessho, T. Kojimoto, and M. Kurata, J. Rheol., 23, 617 (1979).

5. H. Janeschitz-Kriegl, "Polymer Melt Rheology and Flow Birefringence," Springer-Verlag, Berlin, 1983, Chapter 2.

6. A. S. Lodge, Trans. Faraday Soc., 52, 120 (1956).

7. W. Philippoff, J. Appl. Phys., 27, 984 (1956).

8. J. L. S. Wales, "The Application of Flow Birefringence to Rheological Studies of Polymer Melts," Delft Univ. Press, 1976.

9. M. Doi and S. F. Edwards, "The Theory of Polymer Dynamics," Clarendon Press, Oxford, 1986, Chapter 7.

10. E. Pierri and A. Dondos, Eur. Polym. J., 23, 347 (1987).

11. P. L. Frattini and G. G. Fuller, J. Rheol., 28, 61 (1984).

12. J. S. Lee and G. G. Fuller, J. Non-Newtonian Fluid Mech., 26, 57 (1987).

13. W. A. Shurcliff, "Polarized Light," Harvard Univ. Press, 1962, Chapter 8.

14. Z. Hashin, in "Second-Order Effects in Elasticity Plasticity and Fluid Dynamics," M. Reiner and D. Abir, Ed., McMillan, New York, N. Y., 1964.

15. R. F. Heitmiller, R. Z. Maar, and H. H. Zabusky, J Appl. Polym. Sci., 8, 873 (1964).

16. K. Osaki, E. Takatori, M.Kurata, H. Ohnuma, and T. Kotaka, Polym. J., 18, 947 (1986).

17. R. G. Larson, S. A. Khan, and V. R. Raju, J. Rheol., 32, 145 (1988).

18. D. I. Livingston and J. E. Brown, 5th Proc. Int Congr. Rheol., Vol. 4, Uiv. Tokyo Press, 1970. 